\title{
PRODUÇÃO, ACÚMULO E DECOMPOSIÇÃO DE SERAPILHEIRA EM UMA ÁREA REVEGETADA DO PARQUE ESTADUAL MASSAIRO OKAMURA EM MATO GROSSO
}

\author{
C. M. A. VALENTINI ${ }^{*}$, G. S. SOARES, R. A. SANTANA, A. F. S. GUIMARÃES e A. H. B. SILVA \\ Instituto Federal do Mato Grosso - IFMT \\ carla.valentini@blv.ifmt.edu.br ${ }^{*}$
}

Artigo submetido em abril/2013 e aceito em novembro/2014

DOI: $10.15628 /$ holos.2014.1397

\section{RESUMO}

Estimou-se a produção e a taxa de decomposição de serapilheira em uma área revegetada do Instituto Federal de Educação Ciência e Tecnologia do estado de Mato Grosso - IFMT, Campus Cuiabá-Bela Vista, pertencente ao Parque Estadual Massairo Okamura, durante o período de dezembro de 2009 a agosto de 2011. Nos 21 meses de observação constatou-se uma produção média mensal de serapilheira de 990,57 Kg. ha-1 e o acúmulo médio mensal foi de $10957,31 \mathrm{Kg}$.ha-1. A composição média de serapilheira produzida mostrou-se em 43,98\% de folhas; $23,05 \%$ de galhos; $17,64 \%$ de sementes e $16,95 \%$ de outros e para a quantidade média acumulada resultou-se em $41,92 \%$ de folhas; $32,04 \%$ de galhos; $17,22 \%$ de sementes e $14,40 \%$ de outros. A taxa de decomposição média da serapilheira foi de 1,004 e o tempo médio de renovação da quantidade de serapilheira acumulada sobre o solo foi de 11,94 meses. O tempo médio necessário para o desaparecimento de 50 e $95 \%$ da serapilheira foi, respectivamente de 8,28 meses e 35,84 meses. Constatou-se que a deposição de serapilheira encontrada neste estudo demonstrou a importância desta via de ciclagem de nutrientes para manutenção da produtividade das espécies revegetadas no local e para a recuperação da área degradada.

PALAVRAS-CHAVE: serapilheira, áreas degradadas, Cerrado, reflorestamento, conservação.

\section{LITTER PRODUCTION, ACCUMULATION AND DECOMPOSITION IN A STATE PARK AREA REVEGETATED MASSAIRO OKAMURA IN MATO GROSSO}

\begin{abstract}
We estimated the production rate of decomposition of litter in an area replanted Federal Institute of Education Science and Technology of the state of Mato Grosso IFMT Campus Cuiabá-Bela Vista belonging to the State Park Massairo Okamura during the period December 2009 to in August 2011. In the 21 months of observation there was an average monthly output of litter of 990.57 Kg.ha-1 and the average monthly accumulation was 10957.31 Kg.ha-1. . The average composition of litter was found to be produced in $43.98 \%$ of sheets, $32.04 \%$ of branches, $17.64 \% \%$ of seeds and $16.95 \%$ of others and to the average amount accumulated resulted in $41.92 \%$
\end{abstract}

of leaves, $32.04 \%$ of branches, $17.22 \%$ of seeds and $14.40 \%$ of others. The average rate of decomposition of litter was 1.004 and the average time of renewal of the amount of litter accumulated on the soil was 11.94 months. The average time required for the disappearance of 50 and $95 \%$ of the litter was, respectively, of 8.28 months and 35.84 months. It was found that the deposition of litter found in this study demonstrated the importance of this route of nutrient cycling to maintain the productivity of the species at the site revegetated and recovery of degraded areas.

KEYWORDS: litter, degraded areas, Savannah, reforestation, conservation. 


\section{INTRODUÇÃO}

O Cerrado foi incluído recentemente como um dos hotspots para a conservação da biodiversidade mundial (KLINK \& MACHADO, 2005), pela sua alta biodiversidade e alto grau de endemismo, mas também por seu baixo nível de proteção (FIEDLER et al., 2004). MACHADO et al. (2004) estimaram que, se mantidas as taxas de desmatamento relatadas (1,1\% ou 2,2 milhões de hectares de perda anual), o Cerrado deve desaparecer por volta de 2030. É preciso considerar que os recursos naturais oferecidos por ele, uma vez extintos, estarão indisponíveis às futuras gerações e uma das ferramentas para preservá-lo, dada a escala de alteração e degradação já ocorridas, deveria ser a execução de ações que fortaleçam as áreas protegidas já existentes e que criem novas áreas de proteção (KLINK \& MACHADO, 2005).

Em Mato Grosso este bioma corresponde a aproximadamente $40 \%$ da área do estado, concentradas em duas regiões principais: o Pantanal de Mato Grosso e o trecho Depressão Cuiabana - Chapada dos Guimarães (IVANAUSKAS et al., 2004).

Na cidade de Cuiabá, com o intuito de preservar a biodiversidade de uma área de 86,14 hectares de Cerrado no perímetro urbano, que além de uma fauna e flora riquíssima, ainda abriga as nascentes do córrego Barbado e do Moinho, em 1989 foi criada a Reserva Ecológica Massairo Okamura, Unidade de Conservação, categoria bem restritiva que não permitia visitas. Porém o próprio Governo do Estado efetuou doações de partes desta Reserva para órgãos púbicos, como foi o caso da Escola Técnica Federal de Mato Grosso (ETF-MT), hoje IFMT campus Cuiabá-Bela Vista que na época foi contemplada com $144.000 \mathrm{~m}^{2}$ (CAMPOS \& SILVA, 1998).

Quando a Reserva Massairo Okamura passou a categoria de Parque Estadual em 2001, começando assim a construção de infra-estrutura para receber a população, já estava com 53 hectares, ou seja, perdeu $38,47 \%$ de sua área por causa das ocupações oficiais e "clandestinas".

Com a ocupação desta área recebida pela Instituição parte de sua cobertura vegetal original foi perdida, e em 1998 deu-se início a um processo de recuperação do local por revegetação com espécies nativas do cerrado, especialmente as frutíferas, como a embaúba, jatobá, jenipapo, cumbarú, etc., visando a manutenção alimentar da fauna residente ou utilitária dessa área (CAMPOS \& SILVA, 1998).

Uma das formas de avaliar e monitorar o grau de degradação/recuperação de um ecossistema é o uso da serapilheira como bioindicador ambiental, sendo um dos métodos mais utilizados, em escala nacional, para avaliação de reflorestamentos com fins de recuperação ambiental (MOREIRA et al., 2004; ARAÚJO, 2005).

A serapilheira é representada pelo material morto, proveniente da biomassa aérea da vegetação, que permanece no solo até ser fragmentado e decomposto por processos físicoquímicos e bióticos que ocorrem nesta importante fração do ecossistema (ADUAN, 2003). É a principal via de transferência de elementos da vegetação para o solo (XU \& HIRATA, 2002), visto que quantidades baixas de nutrientes entram através da chuva ou do intemperismo do solo (KÖNIG et al., 2002). Através da sua produção é possível estimar a dinâmica de produção de vegetação assim como sua dinâmica de crescimento (UNESCO, 1978). 
A natureza e magnitude das estações climáticas podem interferir na produção de serapilheira, seu acúmulo no solo, e valores de retorno e de sua decomposição, podendo ser consideravelmente diferentes de ano em ano (WIEDER \& WRIGHT, 1995).

O objetivo deste trabalho foi monitorar a produção e decomposição de serapilheira de uma área revegetada do IFMT-campus Cuiabá Bela Vista, após uma década e meia de crescimento das espécies, de forma a avaliar o papel da serapilheira como indicador de recuperação de uma área degradada.

\section{MATERIAL E MÉTODOS}

\section{1 Área de Estudo}

O estudo foi conduzido em uma área revegetada de $600 \mathrm{~m}^{2}$ do Instituto Federal de Educação Ciência e Tecnologia do estado de Mato Grosso - IFMT, Campus Cuiabá-Bela Vista (1534'45,02"S e 5603'45,78'O), pertencente ao Parque Estadual Massairo Okamura durante o período de dezembro de 2009 a agosto de 2011.

No levantamento florístico do local foram amostrados 181 indivíduos, 18 famílias botânicas e 32 espécies As espécies que apresentaram os maiores índices de valor de cobertura (IVC) de foram a Jacaranda cuspidifolia Mart (20,2\%) e a Anadenanthera macrocarpa (Benth) Brenan $(12,3 \%)$, ou seja, boa parte da serapilheira que cobre o solo do local e colabora na ciclagem de nutrientes corresponde ao material destas espécies (MORAIS et al., dados não publicados).

Na composição granulométrica do solo foi encontrado $62 \%$ de areia, $22 \%$ de silte e $16 \%$ de argila, o pH foi igual a 5 e matéria orgânica $36 \mathrm{~g} . \mathrm{dm}^{-3}$ (VALENTINI, 2009).

O clima da região é do tipo Aw segundo a classificação de Köppen, ou seja, tropical semiúmido, sendo a principal característica desse regime térmico a presença constante de temperaturas elevadas, registrando média anual em torno de $25^{\circ}$ a $32^{\circ} \mathrm{C}$, com duas estações bem definidas: uma seca (outono-inverno) e uma chuvosa (primavera-verão). O índice pluviométrico anual varia de 1250 a $1500 \mathrm{~mm}$ (MAITELLI, 1994). Mais de 70\% do total de chuvas acumuladas durante o ano se precipitam de novembro a março. $O$ inverno é excessivamente seco, pois as chuvas são muito raras.

\subsection{Produção de serapilheira}

Para determinação da produção de serapilheira foram utilizados 10 coletores confeccionados com tela de nylon de $2 \mathrm{~mm}$ de abertura de malha e com de $1 \mathrm{~m}^{2}$ de área. Os coletores foram distribuídos de forma aleatória na área de estudo e colocados à 0,3 $\mathrm{m}$ acima do solo para evitar perdas de serapilheira pela ação de microrganismos. (Figura 1).

\subsection{Acúmulo de serapilheira}

Para a determinação do estoque no solo coletaram-se 10 amostras de serapilheira em quadrantes de $25 \times 25 \mathrm{~cm}$ nas proximidades de cada coletor de serapilheira (Figura 1). 
A serapilheira interceptada pelos coletores suspensos (serrapilhera produzida ou litter fall) assim como amostras de serapilheira acumulada sobre o solo (litter pool) foram coletadas mensalmente durante o período de dezembro de 2009 a agosto de 2011.

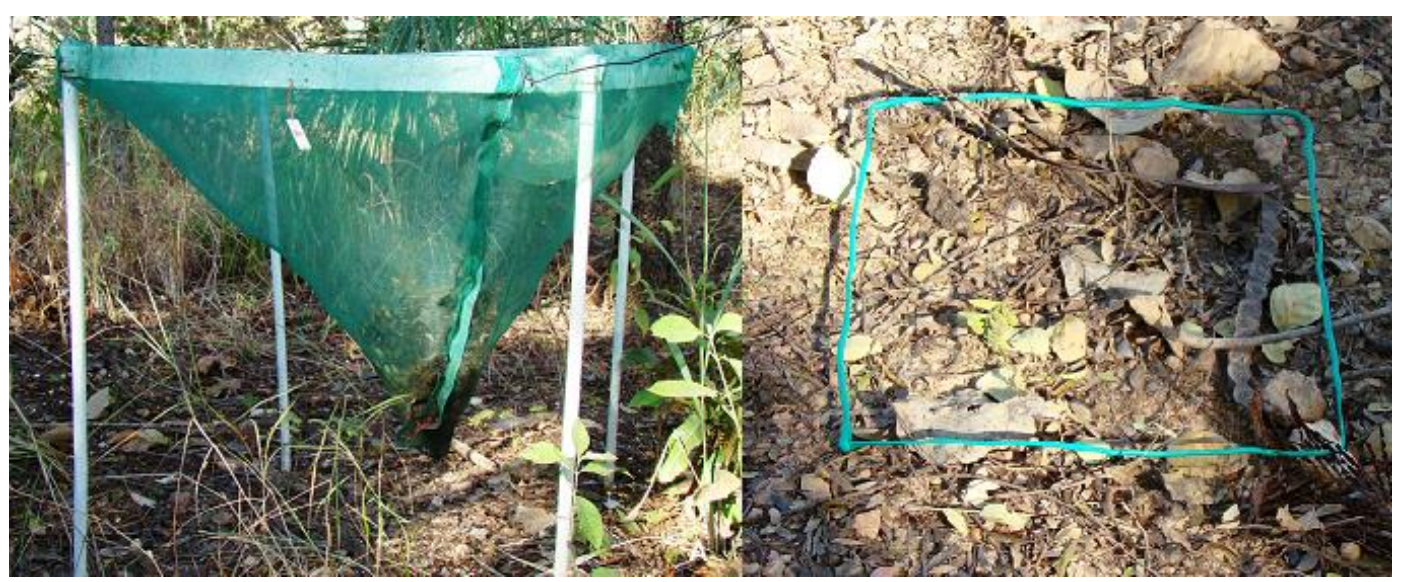

Figura 1. À esquerda coletor de serapilheira produzida e à direita quadrante de coleta de serapilheira acumulada. (Fonte: Arquivo Pessoal)

Vale ressaltar que o experimento foi interrompido 3 meses antes do previsto, que seria em novembro de 2011, devido a um incêndio ocorrido no mês de agosto de 2011 no local, queimando parte dos coletores.

Cada amostra coletada foi separada em frações: folhas, galhos, sementes e miscelânea (materiais que não se conseguiu identificar e materiais de origem animal). Em seguida foram embaladas em sacos de papel, levadas à de circulação forçada a $65^{\circ} \mathrm{C}$ durante $72 \mathrm{~h}$ e pesadas em pesadas em balança de precisão de 0,001g.

\subsection{Taxa de decomposição da serapilheira}

A taxa de decomposição da serapilheira foi estimada a partir da equação proposta por OLSON (1963):

$$
K=\frac{L}{X s s}
$$

$\mathrm{Na}$ equação (1), com valores dados em $\left(\mathrm{g} / \mathrm{m}^{2}\right)$, L representa a quantidade de serapilheira produzida anualmente, Xss a média anual de serapilheira acumulada sobre o solo e $\mathrm{K}$ a constante de decomposição na condição de equilíbrio dinâmico.

Calculou-se também, a partir do valor $K$, o tempo médio de renovação, estimado por $1 / K$.

Foram calculados ainda os tempos necessários para o desaparecimento de 50 e 95\% da serapilheira, segundo SHANKS \& OLSON (1961):

$$
\begin{aligned}
& T 0,5=\frac{0,693}{K} \\
& T 0,05=\frac{3}{K}
\end{aligned}
$$




\subsection{Dados meteorológicos}

Os dados meteorológicos de temperatura média e índice pluviométrico foram obtidos do CPTEC- INPE (Instituto Nacional de Pesquisas Espaciais) e INMET (Instituto Nacional de Meterologia) disponíveis no site da Agritempo (http://www.agritempo.gov.br/agroclima /sumario?uf=MT).

\section{RESULTADOS E DISCUSSÃO}

\subsection{Condições climáticas}

Na Figura 2 são apresentados os dados médios de temperatura do ar e precipitação acumulada mensal, no período estudado.

O clima da região é classificado em três períodos, segundo DUARTE (2000), em função da temperatura, destacando-se uma estação seca e mais fresca no inverno; uma estação de transição seca e mais quente, um pouco antes das chuvas e, uma estação úmida e quente, durante as chuvas do verão. No período chuvoso, principalmente nos primeiros meses do ano, a temperatura é mais elevada. O período seco estende-se de junho a setembro, havendo alguns anos com períodos mais pronunciados. É no período seco que ocorrem as frentes frias, ocasionadas pelo avanço das massas de ar vindas dos pampas argentinos, situados mais próximos das geleiras do Pólo Sul. $O$ frio geralmente acompanha-se de garoas persistentes empurradas para o norte por ventos intensos do sul; permanece, no máximo, oito dias, para reaparecer a intervalos mais ou menos prolongados (BOMBLED, 1976)

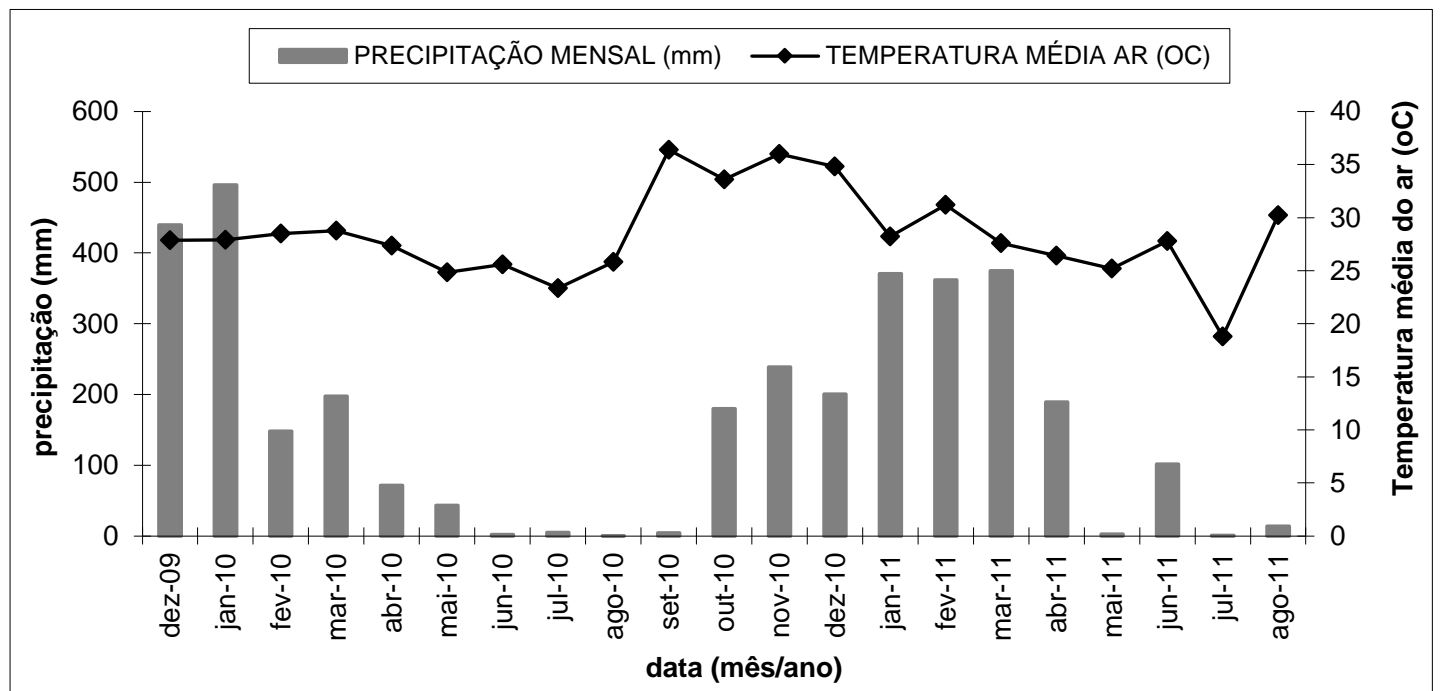

Figura 2. Temperaturas médias e precipitação acumulada no período de dezembro de 2009 a agosto de 2011 no local de estudo.

Observou-se que no período chuvoso de 2009/2010, as chuvas de dezembro e janeiro foram bem maiores que no mesmo período 2010/2011, porém o comportamento se inverteu nos meses de fevereiro, março e abril onde a precipitação foi maior no mês de 2011 que em 2010. A 
seca em 2010 também foi maior do que em 2011, sendo que neste último ano ocorreu atipicamente chuvas intensas no mês de junho $(101 \mathrm{~mm})$, considerado um mês seco na região.

\subsection{Aporte de serapilheira e comportamento sazonal}

Nos 21 meses de observação constatou-se uma produção média mensal de serapilheira de 990,57 Kg. ha-1 e o acúmulo médio mensal foi de 10957,31 Kg.ha-1

A composição média de serapilheira produzida mostrou-se em 43,98 \% de folhas, $23,05 \%$ de galhos, $17,64 \%$ de sementes e $16,95 \%$ de outros.

Considerando a massa total, observou-se que a maior massa de serapilheira produzida ocorreu na época de seca, principalmente nos meses de julho e agosto de 2010 e 2011 (Figura 3), onde registou-se as menores temperaturas médias do ar e precipitação acumulada próxima de zero (Figura 2). O maior aporte de serapilheira no final da estação seca pode ser ocasionado pelo estresse hídrico ocorrido nos meses anteriores, promovendo o aumento nos níveis endógenos dos promotores da senescência, etileno e ácido abscísico, resultando na queda das folhas (DIAS et al., 2002). É de conhecimento que diversas espécies do cerrado são caducifólias, sendo que este evento ocorre no período da seca. Cesar (1991) observou maior queda de folhas nas ocasiões em que ocorreram maiores deficiências hídricas do solo, sendo que a correlação entre maior produção de serapilheira com a menor disponibilidade de água no solo foi constatada, segundo o autor, em vários estudos, para diferentes formações vegetais. Esta maior produção no período seco também pôde se observada por SILVA et al. ( 2007) nas áreas de cerrado senso stricto, cerradão e Floresta de Transição no norte de Mato Grosso, por CAMPOS et al.(2008) em estudo realizado em fragmento de Cerrado stricto senso em Minas Gerais.

As folhas também foram o componente principal produzido da massa seca no período citado, como também verificado no estudo de SANCHES et al. (2008) na Floresta de Transição no norte de Mato Grosso. Durante os meses quentes de verão, quando as chuvas se concentram e os dias são mais longos, o cerrado é muito verde. No inverno, ao contrário, o capim amarelece e seca; quase todas as árvores e arbustos, por sua vez, trocam a folhagem senescente por outra totalmente nova (MARIOT et al., 2008).

Interessante notar que as sementes tiveram uma produção maior no mês de outubro, o que possivelmente indica a época de reprodução das espécies que foram plantadas no local (Figura 3). Os picos que ocorreram no final do período seco e início do chuvoso podem garantir e ampliar a germinação de sementes, visto que a dispersão acontece em época favorável ao estabelecimento e crescimento de plântulas, aumentando o sucesso reprodutivo das espécies (MARQUES, 2002) e garantindo a manutenção da diversidade de espécies na comunidade.

Para a quantidade média acumulada resultou-se em 41,92 \% de folhas, 32,04 \% de galhos, $17,22 \%$ de sementes e $14,40 \%$ de outros. 

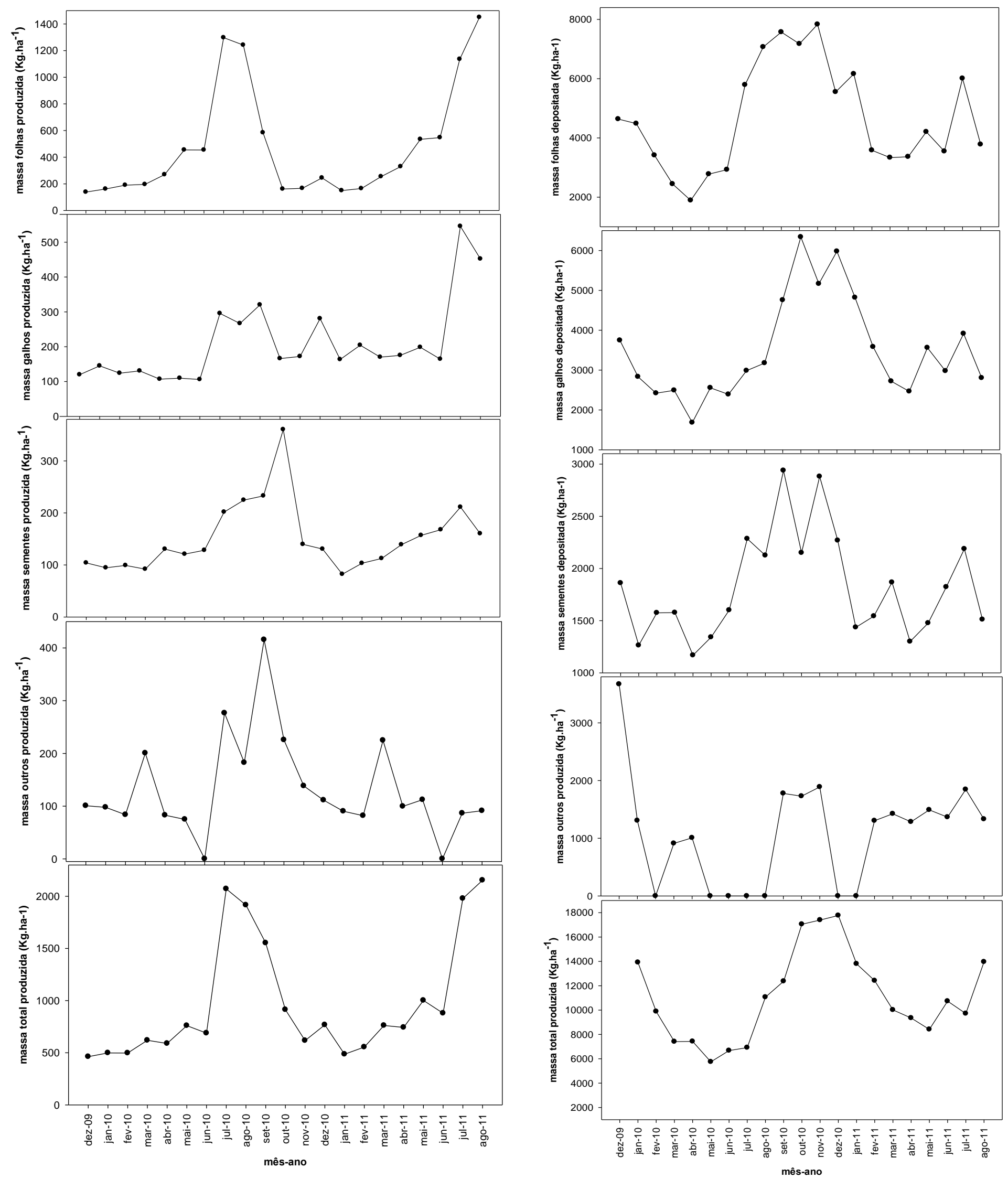

Figura 3. Massa seca produzida de serapilheira na área de estudo.

Figura 4. Massa seca acumulada de serapilheira na área de estudo. 
O maior acúmulo de matéria seca no solo aconteceu nos meses de outubro de novembro de 2011, estação de transição seco-chuvosa, antes das primeiras chuvas na região (Figura 4). O seu acúmulo na superfície do solo é regulado pela quantidade de material que cai da parte aérea das plantas e sua taxa de decomposição. Quanto maior o aporte da serapilheira e quanto menor a sua velocidade de decomposição, maior será a camada de serapilheira acumulada (HAAG, 1985). Estudos em invertebrados do solo mostram que, em estações secas, os movimentos da fauna do solo são mais profundos (PRIETO et al., 1999).

A diminuição do material depositado após o inicio das chuvas, que diminuiu consideravelmente nos meses de maior precipitação no local, pode ser explicado pela maior taxa de decomposição pelos microrganismos presentes no solo que com a umidade encontram condições favoráveis para maior degradação da matéria orgânica depositada (Figuras 2 e 4).

De acordo com Toledo (2003) é importante a compreensão dos mecanismos que regulam esse processo dinâmico, onde a entrada de material através da deposição e a "saída" ou transformação via decomposição, acontecem quase simultaneamente, pois corresponde a uma das etapas mais importante da ciclagem de nutrientes em um bioma (HAAG, 1985).

\subsection{Taxa de decomposição de serapilheira}

A taxa de decomposição médio da serapilheira ( $k$ ) foi de 1,004, que indiretamente representa a velocidade com que os nutrientes, ligados a ela, tornam-se disponíveis. $\mathrm{O}$ rápido desaparecimento da serapilheira na área estudada indica rápida ciclagem de nutrientes da vegetação. Portanto, pode-se sugerir que através da decomposição da serapilheira, a vegetação local, de aproximadamente 15 anos, vem contribuindo para a recuperação da fertilidade do solo, principalmente da camada superficial.

Os valores calculados para o tempo médio de renovação da quantidade de serapilheira acumulada sobre o solo e o tempo necessário para o desaparecimento de 50 e $95 \%$ da serapilheira, encontram-se na Tabela 1.

Tabela 1. Taxa de decomposição (K), tempo médio de renovação (1/K) e tempo necessário para decomposição de 50 e $95 \%$ da serapilheira

\begin{tabular}{c|c|c|c}
\hline Taxa de decomposição (K) & $1 / \mathrm{K}$ (anos) & T0,5 (anos) & T0,05 (anos) \\
\hline 1,004 & 0,99 & 0,69 & 2,99 \\
\hline
\end{tabular}

O tempo médio de renovação da quantidade de serapilheira acumulada sobre o solo (1/k) foi de 11,95 meses e o tempo médio necessário para o desaparecimento de 50 e $95 \%$ da serapilheira foi, respectivamente, de 8,28 meses e 35,84 meses. As diferentes frações da serapilheira têm estrutura e composição química distintas e, portanto, decompõem-se em diferentes velocidades. Assim, a velocidade global de decomposição dependerá também da proporção relativa dos diferentes componentes presentes (CIANCIARUSO et al., 2006). O fato de observar-se pouco estoque de serapilheira acumulada na superfície do solo e também pelos resultados apresentados pode-se inferir que no local há uma rápida velocidade de decomposição e, consequentemente, rápido aproveitamento de nutrientes por parte da vegetação, o que favorece a ciclagem e o equilíbrio desse ecossistema. 


\section{CONCLUSÕES}

- A deposição de serapilheira encontrada neste estudo demonstrou a importância desta via de ciclagem de nutrientes para manutenção da produtividade das espécies revegetadas no local.

- A dinâmica da deposição, acumulação e decomposição da serapilheira variaram entre anos em função da distribuição da chuva, em resposta a mecanismos fisiológicos inerentes às espécies usadas na revegetação da área estudada.

- Para obter sucesso na aplicação desse bioindicador em projetos de revegetação, deve-se procurar definir parâmetros que permitam comparar o comportamento das espécies dentro de um ecossistema estável e conservado, primordiais para se estabelecerem critérios e monitorar programas de recuperação de áreas degradadas.

- A restauração dos ecossistemas degradados pode ser um instrumento para a formação de corredores que venham a unir fragmentos remanescentes, como é o caso do Parque Estadual Massairo Okamura, permitindo assim a continuidade do fluxo gênico, necessário para a manutenção das espécies e da variabilidade de suas populações.

\section{AGRADECIMENTOS}

Agradecemos a Fapemat pelo apoio no primeiro ano da pesquisa, a Brindes Excelentes pela doação dos materiais e confecção dos coletores de litter fall e aos alunos que voluntariamente trabalharam no segundo ano da pesquisa.

\section{REFERÊNCIAS BIBLIOGRÁFICAS}

1. ADUAN, R. E. Respiração de solos e ciclagem de carbono em cerrado nativo e pastagem no Brasil Central. 2003. 149f. Tese (Doutorado em Ecologia) - Instituto de Ciências Biológicas, Departamento de Ecologia, Universidade de Brasília, 2003.

2. ARAÚJO, R.S. Chuva de sementes e deposição de serrapilheira em três sistemas de revegetação de áreas degradadas na Reserva Biológica de Poços das Antas, Silva Jardim, RJ. 2002. 102 f. Dissertação (Mestrado em Ciências) - Ciências Ambientais e Florestais, Universidade Federal Rural do Rio de Janeiro, 2005.

3. BOMBLED, J. Meio século de meteorologia. Gráfica Universitária, Universidade Federal de Mato Grosso, Cuiabá, 1976. 74p.

4. BRAY, J.R. \& GORHAM, E. Litter production in forests of the world. Advances in Ecology Research, v. 2, p. 101-157, 1964.

5. CAMPOS, N. B.; SILVA,E.G. Projeto de recuperação de área degradada na reserva ecológica "Massairo Okamura" em Cuiabá-MT. 1998.18p.

6. CESAR, O. Produção de serapilheira na mata mesófila semi decídua da fazenda Barreiro

7. Rico, município de Anhembi, SP. Revista Brasileira de Biologia, v.53, n. 4, p. 671-681, 1991.

8. CIANCIARUSO, M. V., PIRES, J. S., DELITTI, W. B., SILVA, E. F. Produção de serapilheira e decomposição do material foliar em um cerradão na Estação Ecológica de Jataí, município de Luiz Antônio, SP, Brasil. Acta Botanica Brasilica, v.20, n.1, p. 49-59, 2006. 
9. DIAS, H. C. T.; SILVEIRA, V.; FONTES, M.A. L.; OLIVEIRA-FILHO, A. T.; SCOLFORO, J. R. S. Variação temporal de nutrientes na serapilheira de um fragmento de floresta estacional semidecidual montana em Lavras, MG. Revista Cerne, v.8, n.2, p.1-16, 2002.

10. DUARTE, D. H. S. Padrões de ocupação do solo e microclimas urbanos na região de clima tropical continental. 2000.278f. Tese (Doutorado em Arquitetura). Faculdade de Arquitetura e Urbanismo da Universidade de São Paulo. São Paulo, 2000.

11. FIEDLER, N.C.; AZEVEDO, I. N. C.; REZENDE, A. V.; MEDEIROS, M. B.; VENTURIOLI, F. Efeito de incêndios florestais na estrutura e composição florística de uma área de cerrado sensu stricto na fazenda Água Limpa-DF. Revista Árvore, v. 28, n. 1, p. 129-138, 2004.

12. HAAG, H. P. Ciclagem de nutriente em florestas tropicais. Campinas: Fundação Cargill, $1985,114 p$.

13. IVANAUSKAS, N. M.; MONTEIRO, R.; RODRIGUES, R. R. Composição florística de trechos florestais na borda sul-amazônica. Acta Amazônica, v.34, n.3, 2004.

14. KLINK, C.A.; MACHADO, R. Conservation of the Brazilian Cerrado. Conservation Biology, v.19, p.707-713, 2005.

15. KÖNIG, F. G; BRUN, E. J.; SCHUMACHER, M. V.; LONGHI, S. J. Devolução de nutrientes via serrapilheira em um fragmento de floresta de Santa Maria, RS. Brasil Florestal, n. 74, p.45-52, 2002.

16. MACHADO, R.B., RAMOS NETO, M.B., PEREIRA, P.G.P., et al. Estimativas de perda da área do Cerrado brasileiro. Conservation International, Brasília, DF, Brasil, 2004.

17. MAITELLI, G.T. Uma Abordagem Tridimensional de Clima Urbano em Área Tropical Continental: o exemplo de Cuiabá-MT. Tese de Doutorado, Departamento de Geografia, Universidade de São Paulo, 1994, 200p.

18. MARIOT, A. ; MARTINS, L. C.; VIVIANI, R. G.; PEIXOTO, E. R.A utilização de técnicas nucleadoras na restauração ecológica do canteiro de obras da UHE Serra do Facão, Brasil. Disponível em <http://www.cadp.org.ar/docs/congresos/2008/76.pdf>, 2008. Acesso em 05/12/2011.

19. MARQUES, M. C. M. Dinâmica da dispersão de sementes e regeneração de plantas da planície litorânea da Ilha do Mel, Paraná. 2002. 159f. Tese (Doutorado em Ciências Biológicas) Universidade Estadual de Campinas, Campinas, 2002.

20. MOREIRA, P. R.; SILVA, O. A. Produção de serapilheira em área reflorestada. Revista Árvore, v.28, n.1, p.49-59, 2004

21. OLSON, J. S. Energy storage and the balance of producers and decomposers in ecological systems. Ecology, v.44, n.2, p.322-332, 1963.

22. PRIETO, T. D.; VÁZQUEZ, G. M. M.; RODRÍGUEZ, A. C. Comunidades de la mesofauna edáfica en una selva inundable de la Reserva de la Biosfera de Sian Kaan, Quintana Roo, México. Revista de Biologia Tropical, v.47, p.489-492, 1999.

23. SANCHES, L.; VALENTINI, C.M.A.; PINTO JUNIOR, O.B.; NOGUEIRA, J.S.; VOURLITIS, G.; BIUDES, M.S.; SILVA, C.J.; BAMBI, P.; LOBO, F.A. Seasonal and interannual litter dynamics of a tropical semideciduous forest of the southern Amazon Basin, Brazil. Journal of Geophysical Research, v.113, p. 1-9, 2008.

24. SHANKS, R.; OLSON, J.S. First year breakdown of leaf litter in Southern Appalachia Forest 
Science, v.134, p.194-195, 1961.

25. SILVA, C.J.; SANCHES, L.; BLEICH, M.E.; LOBO, F.A.; NOGUEIRA, J.S. Produção de serrapilheira no Cerrado e Floresta de transição Amazônia-Cerrado do centro-oeste brasileiro. Acta Amazônica, v.37, n.4, p. 543-548, 2007.

26. UNESCO, 1978. Tropical Forest Ecosystems: Natural resources research XIV. Paris, 63p. In Ciclagem de nutrientes em florestas tropicais. Haag, H. P. Fundação Cargill, Campinas SP.1985, 144p.

27. VALENTINI, C.M.A. Aspectos etnobotâncios, fenológicos, agronômicos e químicos da Siparuna guianensis Aublet no cerrado de Mato Grosso. 153f. Tese (Doutorado em Agricultura Tropical)- Universidade Federal de Mato Grosso, Cuiabá-MT. 2009.

28. WIEDER, R. K.; WRIGHT, S. J. Tropical forest litter dynamics and dry season irrigation on barro Colorado island, Panamá. Ecology. v.76, n.6, p.1971-1979, 1995.

29. XU, X. N; HIRATA, E. Forest floor mass and litterfall in Pinus luchuensis plantations with and without broad-leaved trees. Forest Ecology and Management. v. 157, p.165-173. 2002. 